\title{
Chemical Constituents of Garcinia parvifolia (Guttiferae)
}

\author{
G.C.L. Ee ${ }^{a^{*}}$, S.H. Ng ${ }^{\text {a }}$,J.K. Goh ${ }^{\text {b }}$, M.A. Sukari ${ }^{a}$ \& M. Rahmani ${ }^{a}$ \\ ${ }^{a}$ Department of Chemistry, Faculty of Science, Universiti Putra Malaysia, \\ 43400, Serdang Selangor, Malaysia. \\ ${ }^{\mathrm{b}}$ School of Arts \& Sciences, Monash University Malaysia, 46150, Petaling Jaya, Selangor \\ * tel: 603-89466785, fax: 603-89435380 gwen@fsas.upm.edu.my (corresponding author) \\ Received $9^{\text {th }}$ July 2007, accepted in revised form $3^{\text {th }}$ August 2008.
}

\begin{abstract}
Detail chemical studies on Garcinia parvifolia have yielded two triterpenoids, stigmasterol (1) and $\beta$-sitosterol (2), three xanthones, 6-deoxyjacareubin (3), daphnifolin (4) and rubraxanthone (5), one benzophenone, isoxanthochymol (6) and one alkaloid, caffeine (7). These compounds were isolated using common chromatographic techniques and were identified by using spectroscopic experiments such as NMR, MS, IR and UV. This is the first report on the isolation of 6-deoxyjacareubin and daphnifolin from Garcinia parvifolia.
\end{abstract}

ABSTRAK Kajian kimia terperinci ke atas Garcinia parvifolia telah menghasilkan dua triterpinoid, stigmasterol (1) dan $\beta$-sitosterol (2), tiga zanton, 6-deoksijacareubin (3), daphnifolin (4) dan rubraxanton (5), satu benzofenon, isoxanthochymol (6) dan satu alkaloid, kafein (7). Struktur sebatian ini ditentukan dengan menggunakan eksperimen spekstroskopi seperti NMR, MS, IR dan UV. Ini adalah kali pertama 6deoksijacareubin dan daphnifolin diperolehi daripada Garcinia parvifolia .

( Garcinia parvifolia, triterpenoids, xanthones, benzophenone)

\section{INTRODUCTION}

The tropical Guttiferae family is well known to be a rich source of isoprenylated xanthones and biflavonoids [1] - [6]. The family of Guttiferae is also known as Clusiaceae and this family comprises 1350 species in 47 genera [7]. The genus Garcinia is best known in Malaysia as a genus of fruit trees. Extracts and pure isolates of Garcinia species have been shown to exhibit significant antimicrobial and pharmacological activities [3],[8]. In continuation of our search for bioactive natural products from Malaysian plants, we examined the stem bark extracts of Garcinia Parvifolia.. We report here the isolation and structural determination of seven compounds (1-7).

\section{MATERIALS AND METHODS}

\section{Plant Material}

The stem bark of Garcinia parvifolia was collected from Sri Aman Sarawak, Malaysia.

\section{General}

Infrared spectra were measured in $\mathrm{KBr} / \mathrm{NaCl}$ pellet on a Perkin-Elmer FTIR Spectrum BX spectrometer. EIMS were recorded on a Shimadzu GCMS-QP5050A spectrometer. NMR spectra were obtained using a Unity INOVA 500 $\mathrm{MHz} \quad \mathrm{NMR} / \mathrm{JEOL} \quad 400 \mathrm{MHz}$ FT NMR spectrometer using tetramethylsilane (TMS) as internal standard. Ultra violet spectra were recorded in $\mathrm{CHCl}_{3}$ on a Shimadzu UV-160A, UV-Visible Recording Spectrophotometer. 


\section{Extraction and Isolation}

The air-dried and powdered stem bark of Garcinia parvifolia $(2.0 \mathrm{~kg})$ was extracted successively with n-hexane, chloroform, ethyl acetate and methanol at room temperature. The extracts were evaporated to dryness under reduced pressure to yield $51.5 \mathrm{~g}$ of crude $\mathrm{n}$ hexane extract, $12.8 \mathrm{~g}$ of crude chloroform extract, $32.6 \mathrm{~g}$ of crude ethyl acetate extract and $63.1 \mathrm{~g}$ of crude methanol extract. The crude nhexane extract of Garcinia parvifolia $(51.5 \mathrm{~g})$ was chromatographed on a vacuum CC silica gel column using a stepwise gradient system (hexane $/ \mathrm{CHCl}_{3}, \quad \mathrm{CHCl}_{3} /$ EtOAc and EtOAc/MeOH) to give 28 fractions (Frs.). Frs. 11-12 were combined and purified in a silica gel mini column to furnish stigmasterol (1) $(15 \mathrm{mg})$ as fine white needles. Frs. 21-23 were combined and subjected to column chromatography $\left(\mathrm{SiO}_{2}\right.$; $\mathrm{n}$-hexane $/ \mathrm{CHCl}_{3}$ gradients) to yield $\beta$-sitosterol (2) $(10 \mathrm{mg})$. Fractionation of the crude chloroform extract of Garcinia parvifolia (12.8 g) over a silica gel column (hexane $/ \mathrm{CHCl}_{3}$, $\mathrm{CHCl}_{3} /$ EtOAc and EtOAc/MeOH gradient) provided 28 fractions. Frs. 13 was further purified by silica gel column $\left(\mathrm{CHCl}_{3} /\right.$ EtOAc and EtOAc/MeOH gradient) to yielded 6deoxyjacareubin (3) $(5 \mathrm{mg})$ after recrystallization from chloroform. Frs. 15-16 were combined and separated over silica gel column $\left(\mathrm{CHCl}_{3} / \mathrm{EtOAc}\right.$ and $\mathrm{EtOAc} / \mathrm{MeOH}$ gradient) to give daphnifolin (4) $(8 \mathrm{mg})$. The crude methanol extract of Garcinia parvifolia $(63.1 \mathrm{~g})$ was fractionated by vacuum column chromatography $\left(\mathrm{SiO}_{2}\right.$; hexane $/ \mathrm{CHCl}_{3}, \mathrm{CHCl}_{3} / \mathrm{EtOAc}$ and $\mathrm{EtOAc} / \mathrm{MeOH}$ gradient) to yield 36 fractions. Frs. 5-6 were combined and purified by repeated $\mathrm{CC}\left(\mathrm{SiO}_{2}\right.$; $\mathrm{CHCl}_{3} / \mathrm{EtOAc}$ and $\mathrm{EtOAc} / \mathrm{MeOH}$ gradient) to give rubraxanthone (5) $(8 \mathrm{~g}) . F r .13$ was purified by repeated $\mathrm{CC}\left(\mathrm{SiO}_{2} ; \mathrm{CHCl}_{3} / \mathrm{EtOAc}\right.$ and $\mathrm{EtOAc} / \mathrm{MeOH}$ gradient) to give isoxanthochymol (6) $(12 \mathrm{mg})$. The crude ethyl acetate extract of Garcinia parvifolia (32.6 g) was subjected to column chromatography $\left(\mathrm{SiO}_{2}\right.$; hexane $/ \mathrm{CHCl}_{3}, \mathrm{CHCl}_{3} / \mathrm{EtOAc}$ and $\mathrm{EtOAc} / \mathrm{MeOH}$ gradient) to give 27 fractions. Frs. 8-9 was rechromatographed on a silica gel column $\left(\mathrm{CHCl}_{3} / \mathrm{EtOAc}\right.$ and $\mathrm{EtOAc} / \mathrm{MeOH}$ gradient $)$ to give caffeine (7) (3 mg).

Stigmasterol (1): White needle, mp $169{ }^{\circ} \mathrm{C}$ ([9] Lit. $\left.170{ }^{\circ} \mathrm{C}\right)$. All spectral data agree with literature values [9].
$\beta$-Sitosterol (2): White crystals, mp 134-136 ${ }^{\circ} \mathrm{C}$ ([9] Lit.136-137 ${ }^{\circ} \mathrm{C}$ ). All spectral data agree with literature values [9].

6-Deoxyjacareubin (3): Yellow amorphous powder, mp 203-209 ${ }^{\circ} \mathrm{C}$ ([10] Lit. 211-213 ${ }^{\circ} \mathrm{C}$.). UV (EtOH) $\lambda_{\max } \mathrm{nm}(\log \varepsilon): 412.5$ (2.5). IR $v_{\max }$ $\mathrm{cm}^{-1}$ (KBr): 3792 (broad OH), 2920 (C-H stretching), $1648(\mathrm{C}=\mathrm{O})$. EI-MS m/z (rel.int.): 310 (14), 295 (100), 281 (1), 147 (16), 107 (1), 77 (1). ${ }^{1} \mathrm{H}$ NMR $\left(400 \mathrm{MHz}, \mathrm{CDCl}_{3}\right): \delta 7.57(1 \mathrm{H}$, $\mathrm{dd}, J=8.3,6.4 \mathrm{~Hz}, \mathrm{H}-8), 7.14(1 \mathrm{H}$, dd, $J=8.3$, $6.4 \mathrm{~Hz}, \mathrm{H}-6), 7.05(1 \mathrm{H}, \mathrm{t}, J=8.3, \mathrm{H}-7), 6.88(1 \mathrm{H}$, $\mathrm{d}, J=10.0 \mathrm{~Hz}, \mathrm{H}-11), 6.13(1 \mathrm{H}, \mathrm{s}, \mathrm{H}-4), 5.49$ $(1 \mathrm{H}, \mathrm{d}, J=10.0 \mathrm{~Hz}, \mathrm{H}-12), 1.35(6 \mathrm{H}, \mathrm{s}, \mathrm{H}-14, \mathrm{H}-$ 15). ${ }^{13} \mathrm{C}$ NMR $\left(100 \mathrm{MHz}, \mathrm{CDCl}_{3}\right): \delta 181.1$ (C-9), 162.4 (C-3), 160.7 (C-1), 151.5 (C-4a), 145.7 (C5), 145.1 (C-10a), 126.9 (C-12), 123.8 (C-7), 121.2 (C-8a), 120.4 (C-6), 114.9 (C-11), 115.6 (C-8), 103.3 (C-9a), 101.4 (C-2), 98.9 (C-4), 78.2 (C-13), 27.9 (C-14), 27.9 (C-15).

Daphnifolin (4): Yellow crystals, mp $239{ }^{\circ} \mathrm{C}$ ([11] Lit. $240-242{ }^{\circ} \mathrm{C}$ ). UV (EtOH) $\lambda_{\max } \mathrm{nm}(\log$ ع): 392.5 (2.5). IR $v_{\max } \mathrm{cm}^{-1}$ (KBr): 3550 (broad $\mathrm{OH}), 2946$ (C-H stretching), $1656(\mathrm{C}=\mathrm{O}), 1498$ $(\mathrm{C}=\mathrm{C}$ aromatic). EI-MS m/z (rel.int.): 274 (69), 259 (11), 245 (7), 231 (100), 202 (8), 147 (7), 136 (15), 121 (1), 101 (10), 93 (2), 65 (6), 51 (8). ${ }^{1} \mathrm{H}$ NMR $\left(400 \mathrm{MHz}, \mathrm{CDCl}_{3}\right): \delta 7.70(1 \mathrm{H}, \mathrm{dd}, J=$ $1.8,8.2 \mathrm{~Hz}, \mathrm{H}-8), 7.26(1 \mathrm{H}, \mathrm{t}, J=8.2 \mathrm{~Hz}, \mathrm{H}-7)$, $7.21(1 \mathrm{H}, \mathrm{dd}, J=1.8,8.2 \mathrm{~Hz}, \mathrm{H}-6), 6.60(1 \mathrm{H}, \mathrm{s}$, $\mathrm{H}-2), 3.94\left(3 \mathrm{H}, \mathrm{s}, 4-\mathrm{OCH}_{3}\right) .{ }^{13} \mathrm{C}$ NMR $(100 \mathrm{MHz}$, $\mathrm{CDCl}_{3}$ ): $\delta 181.2(\mathrm{C}-9), 158.2(\mathrm{C}-1), 153.7$ (C-4a), 152.9 (C-3), 145.4 (C-5), 145.1 (C-10a), 130.3 (C-4), 123.3 (C-7), 120.6 (C-8a), 119.8 (C-6), 115.0 (C-8), 102.9 (C-9a), 93.9 (C-2), 60.1 (4$\left.\mathrm{OCH}_{3}\right)$.

Rubraxanthone (5): Yellow crystals, mp 206-209 ${ }^{\mathrm{O}} \mathrm{C}$ ([4] Lit.205-206 ${ }^{\mathrm{O}} \mathrm{C}$ ). UV (EtOH) $\lambda_{\max } \mathrm{nm}$ (log ع): 432 (0.12), 312 (1.06), 238 (1.60), 209 (1.06). IR $v_{\max } \mathrm{cm}^{-1}(\mathrm{KBr}): 3430$ (broad $\left.\mathrm{OH}\right)$, 2970 (C-H stretching), $1640(\mathrm{C}=\mathrm{O}), 1610(\mathrm{C}=\mathrm{C}$ aromatic), $1466(\mathrm{C}=\mathrm{C}$ aromatic). EI-MS $\mathrm{m} / \mathrm{z}$ (rel.int.): 410 (17), 341 (100), 326 (5), 311 (20), 299 (25), 288 (13), 271 (9), 69 (32), 53 (6). ${ }^{1} \mathrm{H}$ NMR (400 MHz, MeOH): $\delta 6.68(1 \mathrm{H}, \mathrm{s}, \mathrm{H}-5)$, $6.17(1 \mathrm{H}, \mathrm{d}, J=1.8 \mathrm{~Hz}, \mathrm{H}-4), 6.08(1 \mathrm{H}, \mathrm{d}, J=$ $1.8 \mathrm{~Hz}, \mathrm{H}-2), 5.18$ (1H, t, $J=6.4 \mathrm{~Hz}, \mathrm{H}-12), 4.97$ (1H, m, H-16), $4.05(2 \mathrm{H}, \mathrm{d}, J=6.4 \mathrm{~Hz}, \mathrm{H}-11)$, $3.74\left(3 \mathrm{H}, \mathrm{s}, 7-\mathrm{OCH}_{3}\right), 2.02(2 \mathrm{H}, \mathrm{m}, \mathrm{H}-14), 1.97$ $(2 \mathrm{H}, \mathrm{m}, \mathrm{H}-15), 1.79(3 \mathrm{H}, \mathrm{s}, \mathrm{H}-18), 1.53(3 \mathrm{H}, \mathrm{s}$, $\mathrm{H}-19), 1.49$ (3H, s, H-20). ${ }^{13} \mathrm{C}$ NMR (100MHz, $\mathrm{MeOH}): \delta 183.1$ (C-9), 165.9 (C-1), 164.8 (C-3), 158.4 (C-6), 158.1 (C-4a), 156.8 (C-10a), 144.9 
(C-7), 138.7 (C-8), 135.4 (C-13), 132.0 (C-17), 125.5 (C-16), 125.2 (C-12), 112.2 (C-8a), 102.9 (C-5), 103.9 (C-9a), 98.8 (C-2), 94.0 (C-4), 61.4 $\left(7-\mathrm{OCH}_{3}\right), 40.8(\mathrm{C}-14), 27.6(\mathrm{C}-15), 27.0(\mathrm{C}-11)$, 25.8 (C-19), 17.7 (C-20), 16.6 (C-18).

Isoxanthochymol (6): Bright yellow solid, mp of 123-125 ${ }^{\mathrm{O}} \mathrm{C}$ ([12] m.p. $\left.125-127{ }^{\circ} \mathrm{C}\right)$. UV (EtOH) $\lambda_{\max } \mathrm{nm}(\log \varepsilon): 414$ (2.50). IR $v_{\max } \mathrm{cm}^{-1}(\mathrm{KBr})$ : $3428(\mathrm{OH}), 2928$ (C-H stretching), $1728(\mathrm{C}=\mathrm{O})$, $1634(\mathrm{C}=\mathrm{C}), 1444\left(\mathrm{CH}_{2}\right.$ bending $), 1378\left(\mathrm{CH}_{3}\right.$ bending), 1198 (C-O). EI-MS m/z (rel.int.): 602 (1.8), 465 (38), 411 (3), 355 (6), 341 (18), 285 (5), 231 (39), 217 (6), 177 (12), 137 (5), 109 (5), 95 (15), 81 (23), 69 (100), 55 (25), 41 (3). ${ }^{1} \mathrm{H}$ NMR (400 MHz, $\left.\mathrm{CDCl}_{3}\right): \delta 6.97(1 \mathrm{H}, \mathrm{d}, J=8.3$ Hz, H-16), 6.95 (1H, s, H-12), $6.61(1 \mathrm{H}, \mathrm{d}, \mathrm{J}=$ $8.3 \mathrm{~Hz}, \mathrm{H}-15), 5.10$ (1H, m, H-35), 4.95 (1H, m, H-25), 4.80 (1H, m, H-18), 2.74 (2H, m, H-7), 2.63 (1H, m, H-30), 2.38 (1H, m, H-6), 2.19 (1H, m, H-17a), 2.16 (1H, m, H-17b), 2.08 (2H, m, H24), 1.86 (2H, m, H-34), 1.81 (3H, s, H-32), 1.75 (3H, s, H-33), 1.71 (3H, s, H-37), $1.70(3 \mathrm{H}, \mathrm{s}, \mathrm{H}-$ 38), 1.60 (2H, d, $J=6.4 \mathrm{~Hz}, \mathrm{H}-29), 1.56(3 \mathrm{H}, \mathrm{s}$, H-20), 1.54 (3H, s, H-27), 1.48 (3H, s, H-21), 1.45 (3H, s, H-28), 1.19 (3H, s, H-22), 1.02 (3H, s, H-23). ${ }^{13} \mathrm{C}$ NMR (100MHz, $\left.\mathrm{CDCl}_{3}\right): \delta 209.2$ (C-9), 195.0 (C-3), 193.8 (C-10), 149.8 (C-14), 147.2 (C-1), 145.7 (C-36), 143.6 (C-13), 135.3 (C-26), 132.8 (C-19), 127.4 (C-11), 124.0 (C-16), 123.6 (C-25), 119.9 (C-35), 116.4 (C-12), 115.7 (C-15), 114.2 (C-2), 113.3 (C-18), 109.6 (C-31), 69.7 (C-4), 49.6 (C-8), 46.7 (C-5), 43.4 (C-7), 43.2 (C-6), 42.4 (C-30), 36.4 (C-32), 35.3 (C-33), 28.8 (C-17), 26.8 (C-23), 26.2 (C-29), 26.0 (C20), 25.7 (C-27), 25.6 (C-24), 25.6 (C-34), 22.6 (C-22), 22.5 (C-37), 18.1 (C-21), 17.8 (C-28), 17.0 (C-38).

Caffeine (7) - Green crystals, mp $234{ }^{\mathrm{O}} \mathrm{C}$. ([13] m.p. )UV (EtOH) $\lambda_{\max } \mathrm{nm}(\log \varepsilon): 502.5$ (0.05), 495.0 (0.05), 412.5 (0.22), 389.0 (0.21), 296.0 (2.50). IR $v_{\max } \mathrm{cm}^{-1}(\mathrm{KBr}): 3436(\mathrm{~N}-\mathrm{H}), 3112(\mathrm{C}-$ $\mathrm{H}$ stretching), $1704(\mathrm{C}=\mathrm{O}), 1658(\mathrm{C}=\mathrm{C}), 1548$ $(\mathrm{C}=\mathrm{N}), 1484(\mathrm{C}-\mathrm{N})$. EI-MS m/z (rel.int.): 194 (100), 165 (7), 136 (5), 109 (56), 94 (4), 82 (33), 67 (54), 55 (65). ${ }^{1} \mathrm{H}$ NMR (400 MHz, $\left.\mathrm{CDCl}_{3}\right): \delta$ 7.52 (1H, s, H-4), 4.00 (3H, s, H-8), 3.59 (3H, s, $\mathrm{H}-6), 3.41$ (3H, s, H-7). ${ }^{13} \mathrm{C}$ NMR $(100 \mathrm{MHz}$, $\left.\mathrm{CDCl}_{3}\right): \delta 155.4(\mathrm{C}-2), 151.7(\mathrm{C}-1), 148.7$ (C-5), 141.4 (C-4), 107.6 (C-3), 33.6 (C-8), 29.7 (C-7), 27.9 (C-6).

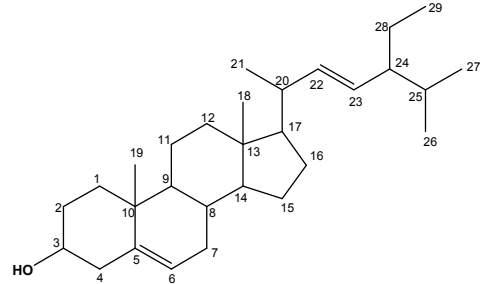

(1)

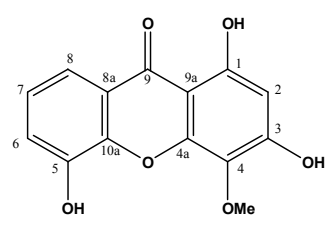

(4)<smiles>Cn1c(=O)c2c(ncn2C)n(C)c1=O</smiles>

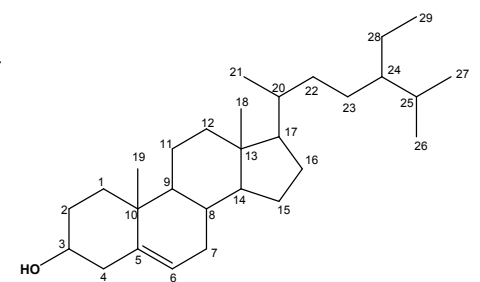

(2)

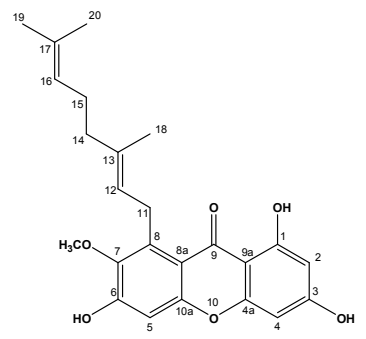

(5)

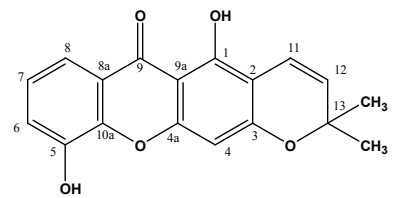

(3)

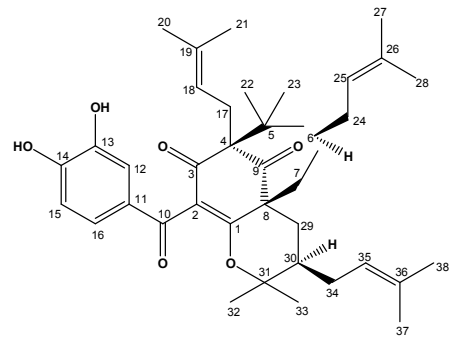

(6) 


\section{RESULTS AND DISCUSSION}

6-Deoxyjacareubin (3) was obtained as yellow amorphous powder after recrystallisation from chloroform, m.p. 203-209 ${ }^{\circ} \mathrm{C}$ ([10] Lit. 211-213 ${ }^{\mathrm{O}} \mathrm{C}$ ). A positive test with alcoholic ferric chloride revealed its phenolic nature. The $\left[\mathrm{M}^{+}\right]$at $\mathrm{m} / \mathrm{z} 310$ in the EI mass spectrum corresponds to the molecular formula $\mathrm{C}_{18} \mathrm{H}_{14} \mathrm{O}_{5}$. The FTIR spectrum exhibited strong bands at $3792 \mathrm{~cm}^{-1}$ and 1648 $\mathrm{cm}^{-1}$ due to a phenolic hydroxyl and a chelated carbonyl group. The UV spectrum of (3) exhibited characteristic absorption bands of a xanthone at $412.5 \mathrm{~nm}$.

The ${ }^{1} \mathrm{H}$ NMR spectrum of (3) indicated the presence of signals at $\delta 7.14(1 \mathrm{H}, \mathrm{dd}, J=8.3 \mathrm{~Hz}$, $6.4 \mathrm{~Hz}), \delta 7.05(1 \mathrm{H}, \mathrm{t}, J=8.3 \mathrm{~Hz})$ and $\delta 7.57(1 \mathrm{H}$, dd, $J=8.3,6.4 \mathrm{~Hz}$ ) which were assigned to H-6, $\mathrm{H}-7$ and $\mathrm{H}-8$ respectively. The occurrence of a doublet doublet at $\delta 7.14$ was due to orthocoupling with $\delta 7.05$ and meta-coupling with $\delta$ 7.57. The H-8 signal was at a lower field as it was deshielded by the carbonyl group at C-9. In addition, the remaining one-proton singlet at $\delta$ 6.13 was attributed to an isolated aromatic proton at H-4. Other than that, signals at $\delta 6.88$ $(1 \mathrm{H}, \mathrm{d}, J=10.0 \mathrm{~Hz}, \mathrm{H}-11), \delta 5.49(1 \mathrm{H}, \mathrm{d}, J=$ $10.0 \mathrm{~Hz}, \mathrm{H}-12)$ and $\delta 1.35\left(6 \mathrm{H}, \mathrm{s}, 2 \mathrm{CH}_{3}\right)$ indicated the presence of a 2,2-dimethyl chromene ring substitution at the xanthone ring, with the chromene double bond ortho to the C-1 hydroxyl group.
The ${ }^{13} \mathrm{C}$ NMR spectrum disclosed the presence of one conjugated carbonyl group at $\delta 181.1$ (C-9) and eight substituted aromatic carbons at $\delta 160.7$ (C-1), $\delta 101.4$ (C-2), $\delta 162.4$ (C-3), $\delta 151.5$ (C4a), $\delta 145.1$ (C-10a), $\delta 145.7$ (C-5), $\delta 121.2$ (C$8 a)$ and $\delta 103.3(\mathrm{C}-9 \mathrm{a})$.

The 18 carbon signals in the ${ }^{13} \mathrm{C}$ NMR spectrum were characterized by the DEPT spectrum, which indicated that (3) consists of two methyl, six methine and ten quaternary carbons.

Conclusive proof for the substitution pattern of (3) came from analysis of the correlation peaks in the HMBC spectra. In the HMBC spectrum, the location of a hydroxyl at C-5 was supported by the observed cross peak between the hydroxyl bearing $\mathrm{C}-5$ and the aromatic proton of $\mathrm{H}-6$ through a ${ }^{3} \mathrm{~J}$ correlation. $\mathrm{A}^{3} \mathrm{~J}$ correlation was also observed between the doublet at $\delta 6.88(\mathrm{H}-11)$ and $\delta 78.2(\mathrm{C}-13)$ and between $\delta 5.49(\mathrm{H}-12)$ and d $101.4(\mathrm{C}-2)$ indicating the signals at $\delta 6.88$ and $\delta 5.49$ to be for $\mathrm{H}-11$ and $\mathrm{H}-12$. The latter confirms the presence of the pyrano ring attached to $\mathrm{C}-2$ and $\mathrm{C}-3$. This assignment was further confirmed by the coupling between $\delta 6.88$ and $\delta 5.49$ as observed in the ${ }^{1} \mathrm{H}-{ }^{1} \mathrm{H} \quad \mathrm{COSY}$ experiment.

Based on these data the compound was therefore assigned 6-deoxyjacareubin (3). The spectral data are summarized in Table 1. 
Table 1. ${ }^{1} \mathrm{H}$ NMR $\left(400 \mathrm{MHz}, \mathrm{CHCl}_{3}\right),{ }^{13} \mathrm{C} \mathrm{NMR}\left(100 \mathrm{MHz}, \mathrm{CHCl}_{3}\right) \& \mathrm{HMBC}$ data for 6-deoxyjacareubin (3)

\begin{tabular}{|c|c|c|c|}
\hline Position & ${ }^{1} \mathrm{H}(\delta)$ & ${ }^{13} \mathrm{C}(\delta)$ & $\begin{array}{l}\text { HMBC } \\
\text { (C-H correlations) }\end{array}$ \\
\hline 1 & - & 160.7 & $\begin{array}{l}103.3(\mathrm{C}-9 \mathrm{a})\left({ }^{3} \mathrm{~J}\right), 160.7(\mathrm{C}-1) \\
\left({ }^{2} \mathrm{~J}\right)\end{array}$ \\
\hline 2 & - & 101.4 & - \\
\hline 3 & - & 162.4 & - \\
\hline 4 & $6.13(1 \mathrm{H}, \mathrm{s})$ & 98.9 & $\begin{array}{l}103.3(\mathrm{C}-9 \mathrm{a})\left({ }^{3} \mathrm{~J}\right), 162.4(\mathrm{C}-3) \\
\left({ }^{2} \mathrm{~J}\right)\end{array}$ \\
\hline 5 & - & 145.7 & $\begin{array}{l}120.4(\mathrm{C}-6)\left({ }^{3} \mathrm{~J}\right), 145.7(\mathrm{C}-5) \\
\left({ }^{2} \mathrm{~J}\right)\end{array}$ \\
\hline 6 & $7.14(1 \mathrm{H}, \mathrm{dd}, J=8.3 \mathrm{~Hz}, 6.4 \mathrm{~Hz})$ & 120.4 & $145.1(\mathrm{C}-10 \mathrm{a})\left({ }^{3} \mathrm{~J}\right)$ \\
\hline 7 & $7.05(1 \mathrm{H}, \mathrm{t}, J=\mathrm{Hz})$ & 123.8 & $\begin{array}{l}145.7(\mathrm{C}-5)\left({ }^{3} \mathrm{~J}\right), 120.4(\mathrm{C}-6) \\
\left({ }^{2} \mathrm{~J}\right)\end{array}$ \\
\hline 8 & $7.57(1 \mathrm{H}, \mathrm{dd}, J=8.3 \mathrm{~Hz}, 6.4 \mathrm{~Hz})$ & 115.6 & $\begin{array}{l}181.1(\mathrm{C}-9)\left({ }^{3} \mathrm{~J}\right), 120.4(\mathrm{C}-6) \\
\left({ }^{3} \mathrm{~J}\right), 145.1(\mathrm{C}-10 \mathrm{a})\left({ }^{3} \mathrm{~J}\right)\end{array}$ \\
\hline 9 & - & 181.1 & - \\
\hline 11 & $6.88(1 \mathrm{H}, \mathrm{d}, J=10.0 \mathrm{~Hz})$ & 114.9 & $\begin{array}{l}78.2(\mathrm{C}-13)\left({ }^{3} \mathrm{~J}\right), 162.4(\mathrm{C}-3) \\
\left({ }^{3} \mathrm{~J}\right)\end{array}$ \\
\hline 12 & $5.49(1 \mathrm{H}, \mathrm{d}, J=10.0 \mathrm{~Hz})$ & 126.9 & $\begin{array}{l}101.4(\mathrm{C}-2)\left({ }^{3} \mathrm{~J}\right), 78.2(\mathrm{C}-13) \\
\left({ }^{2} \mathrm{~J}\right)\end{array}$ \\
\hline 13 & - & 78.2 & - \\
\hline 14 & $1.35(6 \mathrm{H}, \mathrm{s})$ & 27.9 & $\begin{array}{l}27.9(\mathrm{C}-15)\left({ }^{3} \mathrm{~J}\right), 126.9(\mathrm{C}-12) \\
\left({ }^{3} \mathrm{~J}\right), 78.2(\mathrm{C}-13)\left({ }^{2} \mathrm{~J}\right)\end{array}$ \\
\hline 15 & $1.35(6 \mathrm{H}, \mathrm{s})$ & 27.9 & $\begin{array}{l}27.9(\mathrm{C}-14)\left({ }^{3} \mathrm{~J}\right), 126.9(\mathrm{C}-12) \\
\left({ }^{3} \mathrm{~J}\right), 78.2(\mathrm{C}-13)\left({ }^{2} \mathrm{~J}\right)\end{array}$ \\
\hline $4 a$ & - & 151.5 & - \\
\hline $8 \mathrm{a}$ & - & 121.2 & - \\
\hline $9 a$ & - & 103.3 & - \\
\hline $10 \mathrm{a}$ & - & 145.1 & - \\
\hline
\end{tabular}

\section{ACKNOWLEDGEMENTS}

The authors wish to thank $\mathrm{Mr}$ Mohd Johadi Iskandar of the Chemistry Department, UPM for recording NMR spectra and the Malaysian IRPA grant for financial support.

\section{REFERENCES}

1. Xu, Y.J., Lai, Y.H., Imiyabir, Z. and Goh, S.H. (2001). Xanthones from Garcinia parvifolia. Journal of Natural Product 64: 1191-1195.

2. Bennett, G.J. and Lee, H.H., (1989). Xanthones FromGuttiferae. Phytochemistry 28(4): 967-998.

3. Goh, S.H., Jantan, I., Gray, A.I. and Waterman, P.G. (1991). Prenylated Xanthones from Garcinia opaga. Phytochemistry 31(4):1383-1386.

4.Ampofo, S.A. and Waterman, P.G. (1986). Xanthones From Garcinia Species Phytochemistry 25: 2351-2355.
5. Aumond, M.C., Merza, J., Rondeau, D., Dumontet, V., Seraphin, D. and Richomme, P. (2004). Prenylated Xanthones and Tocotrienols from Garcinia virgata. Phytochemistry 65: 2915-2920.

6. Crichton, E.G. and Waterman, P.G. (1979). Manniflavanone, A New 3,8-linked Flavanone Dimer from the Stem Bark of Garcinia mannii. Phytochemistry 18: 1553-1557.

7. Mabberly, D.J. (1987). The Plant Book. A Portable Dictionary of the Higher Plants. Cambridge: Cambridge University Press.

8. Tay, B.Y.P. (1996). Chemical Constituents of Garcinia mangostana, Garcini parvifolia, Garcinia griffitti and their Biological Activities. M.Sc Thesis, Universiti Putra Malaysia, Selangor, Malaysia.

9. Hill, R.A., Kirk, D.N., Makin, H.L.J. and Murphy, C.M. (1991).Dictionary of Steroids. Great Britain: Chapman and Hall.

10.Gottlieb, O.R., Mesquita, A.A.L., Oliveira, G.G.D. and Melo, M.T.D. (1940). Xanthones 
Malaysian Journal of Science 28 (1): 105-110 (2009)

from Kielmeyera spesiosa. Phytochemistry 9: 2537-2544.

11. Ee, G.C.L., Lim, C.K. and Lee, H.L. (2006). Daphnifolin, A New Xanthone from Mesua daphnifolia (Guttiferae). J. Asian Natural Product Research. 8(6): $567-570$.

12. Cheow Y.L. (2005).Chemical Constituents and Biological Activities of Garcinia maingayi and Garcinia parvifolia. MSc Thesis, Universiti Putra Malaysia, Selangor, Malaysia.

13. Pavia, D.L., Lampman, G.M. and Kriz, G.S. (1988). Introduction to Organic Laboratory Techniques $\left(3^{\text {rd }}\right.$ ed.). 62-66. Washington: Saunders College Publishing. 